\title{
PROPUESTA ESTRATÉGICA Y METODOLÓGICA PARA LA GESTIÓN EN EL TRABAJO COLABORATIVO
}

\author{
Kenneth Jiménez González \\ Docente de la Escuela de Administración Educativa \\ Universidad de Costa Rica \\ San José, Costa Rica
}

Recibido12-VIII-2008 • Aceptado 24-IV-09 • Corregido 30-V-2009

\begin{abstract}
Resumen: El articulo expone una propuesta estratégica y metodológica para la gestión en el trabajo colaborativo. Esta es el resultado de una investigación en la que, desde la perspectiva docente y administrativa, se diagnosticó el trabajo colaborativo como una estrategia metodológica. Se realizó en una unidad pedagógica del Sistema Educativo Costarricense. La propuesta recomienda un cambio en el sentido del trabajo en grupo en los centros de enseñanza para pasar del esquema de gestión de trabajo en grupo a un nivel superior, en el que la organización conciba que el trabajo en equipo propicia la conformación de un sistema colaborativo en el cual cada miembro es importante, lo que se refleja directamente en un clima organizacional armonioso y propenso al mejoramiento continuo, tanto por parte del centro educativo como del personal. La propuesta establece un parámetro estandarizado de trabajo para las organizaciones educativas a partir del trabajo colaborativo como estrategia metodológica. Con esta, las personas quienes administren instituciones educativas tendrán la posibilidad de hacer transformaciones oportunas para alcanzar la excelencia en los centros de enseñanza, en el marco de las demandas educativas emergentes en la era de la globalización.
\end{abstract}

Palabras clave: Propuesta estratégica y metodológica, trabajo colaborativo, administración de la educación, organización educativa.

\section{Introducción}

Costa Rica se encuentra en un proceso de cambios que responden a la realidad mundial y al proceso de globalización debido a que ningún país escapa a las implicaciones de la vinculación con la economía externa y el uso del capital mundializado. Por ello, el Estado Costarricense trabaja en la redefinición de sus políticas públicas, en función del cambio inminente.

En el contexto actual, las políticas públicas deben permitir la inclusión, el mejoramiento del recurso humano nacional y el crecimiento profesional, dentro de una dinámica de intercambio de pensamiento. La política educativa es, en ese marco, una herramienta primordial para que el cambio favorezca a la sociedad, pues determina las oportunidades de desarrollo de la ciudadanía y tiene a su cargo la formación de ciudadanos, que debe ser coherente con las necesidades del país. En este sentido, la educación es una institución estratégica llamada a elevar el nivel y la calidad de vida de la población costarricense por medio de cambios que procuren el entendimiento de 


\begin{abstract}
This article presents the "strategic and methodological proposal for the management of collaborative work" that is the result of a diagnostic given by an investigation concerning team work as a methodological strategy in the Pedagogy Unit of Costa Rican Educational System from a teaching and executive perspective. The proposal recommended to make a change to the idea of team work in educational institutions in order to pass from a scheme of team work to a more superior level in which the group feels that team work brings about the formation of a collaborative system where each member is important. As a result, this influences a harmonious organizational environment that will favor the continuous improvement not only of the institution, but also of its staff. The proposal establishes a standardized guideline of work for educational institutions dealing with collaborative work as a methodological strategy that directors could use in order to make suitable changes in search of the layout goals. In this way, excellency will be achieved by the educational institutions.
\end{abstract}

Key words: Strategic and methodological offer, collaborative work, administration of the education, educational organization. la colaboración como una variable social esencial para el desarrollo.

Las nuevas políticas educativas deben enfocarse en la preparación de las jóvenes generaciones para enfrentar con éxito y dignidad los cambios radicales que marcan nuestra civilización; por ello, es necesario brindar, por medio de la experiencia educativa, herramientas que promuevan sistemas colaborativos de convivencia para un adecuado desenvolvimiento social, en los que se reconozca el trabajo con otras personas como un medio fundamental para el logro de objetivos sociales, en el marco de la era de la globalización y de la sociedad del conocimiento y la información.

Es por ello que el objetivo del presente artículo es brindar una alternativa innovadora a la necesidad de establecer ambientes dinámicos y propicios para el aprendizaje de cualquier organización. Todo ello mediante un trabajo colaborativo fundamentado en valores personales y colectivos que se llevan a la práctica por medio de técnicas accesibles y de bajo presupuesto.

\section{El trabajo colaborativo y su importancia}

Uno de los objetivos básicos que se persigue con la utilización del trabajo colaborativo es promover una adecuada formación y un apropiado desempeño laboral a partir del intercambio de ideas y acciones de los miembros implicados en el proceso de gestión de la organización, lo que incluye la promoción de ideas, actividades formativas y propuestas de acción. Por esa razón, se destaca la importancia de aplicar esta estrategia en los centros escolares, partiendo de fundamentos como los indicados por Huertas y Rodríguez (2006), quien hace referencia a que las personas deben ser asignadas o autoasignadas en los grupos de trabajo de acuerdo con habilidades y competencias específicas necesitadas para cumplir una determinada meta bajo la conducción de un 
coordinador; en este contexto, la empatía, la interdependencia y la selección de un líder son elementos de suma relevancia.

La propuesta se fundamenta en principios generales de la administración. Uno de ellos es la comunicación, que debe ser frecuente, fluida y rápida, y parte del hecho de que no basta con aportar ideas, sino que es indispensable justificarlas por medio de criterios válidos, según Chiavenato (2002). En el trabajo colaborativo, todas las aportaciones brindadas por los miembros del equipo deben ser tratadas de forma crítica y constructiva. Asimismo, todos los miembros deben aportar ideas o argumentaciones con la información de base disponible para compartirla de esta forma, los resultados alcanzados no son la sumatoria del trabajo en grupo, sino el reflejo de su cohesión, de modo que cada miembro del grupo asume una responsabilidad individual para la realización de la actividad. Por lo tanto, es necesario ser responsable para el trabajo final y establecer relaciones socio-afectivas positivas y contextos de interdependencia tangibles.

Esto siguiendo una línea como la indicada por Caldeiro y Vizcarra (1999, p. 1), quien plantea:

La propuesta de trabajo colaborativo, entiende la cooperación como una asociación entre personas que van en busca de ayuda mutua en tanto procuran realizar actividades conjuntas, de manera tal que puedan aprender unos de otros. El Aprendizaje Cooperativo se caracteriza por un comportamiento basado en la cooperación, esto es: una estructura cooperativa de incentivo, trabajo y motivaciones, lo que necesariamente implica crear una interdependencia positiva en la interacción alumno-alumno y alumnoprofesor, en la evaluación individual y en el uso de habilidades interpersonales a la hora de actuar en pequeños grupos.

Para que ese proceso del trabajo colaborativo sea exitoso, es necesario manejar premisas válidas sobre lo que acontece en la institución y se deben tomar precauciones acerca de lo que sea predecible. Al respecto, se deben establecer acciones para efectuar las mejoras pertinentes, según la información obtenida. Todo esto porque, como lo indican Caldeiro y Vizcarra (1999), en las organizaciones es posible encontrar elementos positivos y negativos relacionados con el trabajo colaborativo y con la participación de todos los miembros en la gestión del centro de enseñanza, como los siguientes:

\section{Aspectos positivos:}

- $\quad$ Existe un pensamiento proactivo para lograr mejoras totales en el marco de una perspectiva de calidad.

- $\quad$ Se tiene claro lo que se quiere lograr.

- Se muestra interés por escuchar y respetar a los demás con la finalidad de mejorar las relaciones entre el personal.

- Hay un compromiso serio del personal por conseguir un trabajo cooperativo, en el que cada persona realiza su aporte correspondiente.

- Se proporcionan espacios para comunicarse eficazmente en busca de negociar las diferencias individuales.

- Con claridad, se señalan las labores que debe desarrollar cada uno de los miembros del equipo.

- Se identifica la obtención de resultados en sus diferentes niveles.

- Los procesos de trabajo pretenden que las cosas se hagan efectivamente.

\section{Aspectos negativos:}

- Hay una mala integración de funciones y actividades desarrolladas por diferentes personas.

- En ocasiones, las actividades desarrolladas por la organización no se realizan en forma coordinada.

- Los programas planificados en equipo no apuntan hacia un objetivo común a futuro.

- Es difícil identificar al líder que inicia, facilita, aprueba o desaprueba 
nuevas ideas y prácticas en los subgrupos de trabajo, pues hay pocos en el centro educativo.

- $\quad$ Frecuentemente, no se comparten las responsabilidades entre los miembros de la organización.

- Por problemas de manejo de las relaciones personales, no hay una integración oportuna de los equipos de trabajo.

- Regularmente, los equipos de trabajo no logran expresar los pensamientos ni decir los resultados de manera adecuada.

- $\quad$ No se logra establecer una organización del trabajo que posea estructuras definidas en la institución, lo que se convierte en una dificultad difícil de superar porque no hay una institucionalización del equipo.

- No se alcanza un adecuado proceso de comunicación entre las partes, lo que conduce a la monopolización por parte de algún miembro o grupo de aspectos clave; por ejemplo, la toma decisiones y la expresión de opiniones.

- $\quad$ No se promueve un adecuado reconocimiento de logros en la organización.

Según los aspectos positivos y negativos que se identifique es necesario puntualizar algunos acontecimientos no deseables, que pueden suceder en caso de no trabajar la estrategia con una proyección adecuada. Al respecto, se listan algunos de ellos, los cuales pueden presentarse en el corto, mediano y largo plazo:

\section{A corto plazo:}

- Insatisfacción laboral.

- Sentimiento de trabajo en exceso.

- Percepción de objetivos lejanos.

- Discrepancias en el accionar.

- Desconocimiento de acciones requeridas para mejorar.

- Comunicación cortante.
- Aislamiento profesional.

- Falta de éxito en los proyectos.

- Dificultad para encontrar soluciones.

A mediano plazo:

- Proyectos sin conclusión.

- Desinterés general frente a los proyectos nuevos.

- $\quad$ Apatía ante la necesidad de mejoras.

- Sentimientos de insatisfacción.

- Malas relaciones personales.

- Desgano ante los procesos de comunicación asertivos.

- Procesos de planificación incorrectos.

- Discrepancias entre la administración y el personal.

- Lucha de intereses internos.

- Poca solidaridad.

- $\quad$ No se asumen responsabilidades.

\section{A largo plazo:}

- Ninguna apropiación de la institución por parte del personal.

- Pérdida de credibilidad por parte de la administración.

- Cultura organizacional disgregada.

- Pocos intereses compartidos.

- Participación mínima en la elaboración de proyectos.

- Apatía para cooperar.

- Dificultades para proyectarse a la comunidad.

- Poco sentido de integración con los padres de familia.

- $\quad$ Estructuras de trabajo monótonas.

Partiendo del artículo de Glinz (2006), se puede afirmar que para la implementación del trabajo colaborativo es necesario señalar que lo más importante desde este enfoque es que la suma de esfuerzos y la concurrencia de varias personas son preferibles a la habilidad o capacidad 
aislada de cada persona. Un administrador o una administradora de la educación no debe perder de vista su función: crear las condiciones necesarias para la formación de ciudadanos y ciudadanas, según el contexto social donde se desenvuelven; por lo tanto, la organización educativa es en un escenario propicio para el desarrollo de proyectos que promuevan valores y fundamentos democráticos y la equidad.

La Administración de la Educación es la instancia encargada de definir con claridad el camino que seguirá la institución, lo que permite el establecimiento de proyectos concretos en un ambiente de compromiso profesional y ético para el cumplimiento de las labores, es una de las áreas del conocimiento humano que resulta más compleja y desafiante, en donde, según Chiavenato (citado por Alfaro, 1999, p. 48), su tarea fundamental es

interpretar los objetivos por la organización y transformarlos en acción organizacional a través de la planeación, la organización, la dirección y el control desde los esfuerzos realizados en todas las áreas y niveles de la organización, con tal de alcanzar los objetivos de la manera más adecuada a la situación.

Todo administrador o administradora de la educación debe involucrar a todos los miembros de la organización en el establecimiento de los compromisos, lo que hace que las personas se sientan partícipes y útiles en el cumplimiento de objetivos, que es lo que permite el enfoque del trabajo colaborativo.

Partiendo de las premisas de Robbins (1999), la participación de todos los miembros de la organización al establecer los objetivos y participar en la ejecución de los proyectos determinará el éxito de la gestión del administrador educativo, quien propiciará la confianza cuando se tomen decisiones, lo que, a su vez, influirá en el éxito de las acciones que se emprendan en la institución. La participación activa determina la sensación del propio logro y el de todos, lo que promueve el establecimiento de un mejor sistema de convivencia y establece el trabajo colaborativo como una forma diaria de hacer las tareas y cumplir las metas.

La formación de habilidades para el trabajo colaborativo es un requerimiento para los administradores de la educación. Sin embargo, los enfoques han ido variando $\mathrm{y}$, conforme lo hacen, enfatizan en la participación y la implicación de las personas; es decir, que todos los involucrados sean $\mathrm{y}$ se sientan parte de los procesos que se ejecuten, pues esto constituye un principio del trabajo colaborativo, el cual favorece también los cambios que requiere el sistema educativo para atender las necesidades de formación en la actualidad.

Los procesos de cambio en nuestro sistema educativo se enfrentan a grandes retos, particularmente en el campo de la Administración de la Educación, como por ejemplo: implementar mejores sistemas de comunicación, incrementar la participación y el compromiso y establecer respuestas educativas novedosas en atención a las necesidades del contexto.

El trabajo colaborativo es una respuesta apropiada para romper los viejos paradigmas rígidos y burocráticos, pues incluye sistemas que propician la gestión del talento humano y que reconocen que el cambio es inherente a la persona y un reflejo de quienes integran la organización.

La propuesta debe implementarse para alcanzar y mantener el éxito en las organizaciones modernas, ya que éstas requieren talentos prácticamente imposibles de encontrar en un solo individuo; por ello, las nuevas estructuras de las organizaciones (más planas y con menos niveles jerárquicos) demandan una mayor interacción entre las personas, que no puede lograrse con individualismos, sino más bien con una actitud colaborativa, la cual debe promoverse por medio de esta propuesta.

En las organizaciones, el trabajo colaborativo debe ser considerado como un beneficio y no como una obligación, pues se denota como aliciente para el éxito institucional. Como lo indica De Benito (1999), 
en una organización es fundamental un equipo constituido por todos sus miembros.

Desde el nacimiento de la propuesta, se debe considerar el acuerdo básico y se debe establecer que es preponderante trabajar en conjunto; o sea, formar un equipo de trabajo requiere reconocer las ventajas del trabajo colaborativo, en el cual las ventajas recaen en una notable motivación, un considerable compromiso, más ideas, mayor creatividad y mejor comunicación.

El trabajo colaborativo muestra que su fundamento es buscar ser mejores personas y, a partir de esto, se establecen los objetivos finales. En las organizaciones hay dos formas de hacer las tareas, ya sea individualmente (se mantiene la iniciativa y la creatividad) o grupalmente (dos o más personas interactúan para compartir información y tomar decisiones para llegar a un objetivo común), así que estos aspectos deben ser parte de la propuesta.

En primera instancia, la propuesta busca que el personal de la organización pueda conocer las características del trabajo colaborativo, las cuales son, según Caldeiro y Vizcarra (1999, p. 3):

- Una integración armónica de funciones y actividades desarrolladas por diferentes personas.

- Para su implementación, se pretende que las responsabilidades sean compartidas por sus miembros.

- Se necesita que las actividades desarrolladas se realicen en forma coordinada.

- Se requiere que los programas planificados en equipo apunten a un objetivo común.

- Aprender a trabajar efectivamente como equipo demanda su tiempo, dado que es preciso adquirir habilidades $\mathrm{y}$ capacidades especiales necesarias para el desempeño armónico de su labor.

Además, en la propuesta se especifica que entre sus beneficios se incrementa la producción, se mejora la calidad y se aumenta el compromiso. Asimismo, al fomentar el cambio, al integrar personas distintas y al manifestar aportes de ideas valiosas, mejora la autoestima, lo que es reconocido por el personal, pues se proporciona un alto porcentaje de importancia a ítems que se refieren al cumplimiento de roles, comunicación, relaciones, establecimiento de objetivos, lealtad, entre otros. Conjuntamente, se busca enseñar cómo se debe dar la formación de estos equipos de trabajo para que funcionen eficientemente y permitan el desarrollo del trabajo colaborativo entre los participantes de estos.

Para lograr el establecimiento del trabajo colaborativo, siguiendo a Huertas y Rodríguez (2006), en la propuesta se indica que se deben transformar las individualidades en un equipo por medio de la internalización de un aprendizaje colaborativo, en el cual es necesario favorecer un proceso en que se exploren y elaboren aspectos afines a los siguientes conceptos:

$\begin{array}{ll}\text { - } & \text { cohesión } \\ \text { - } & \text { asignación de roles y normas } \\ \text { - } & \text { definición de objetivos } \\ \text { - } & \text { interdependencia }\end{array}$

La cohesión. Se refiere a la atracción que ejerce la condición de ser miembro de un grupo. Los grupos tienen cohesión a medida que ser un miembro de ellos sea considerado algo positivo y los miembros sientan deseos de pertenecer. En las agrupaciones que tienen asignada una tarea, el concepto se puede plantear desde dos perspectivas: cohesión social y cohesión para una tarea. La cohesión social se refiere a los lazos de atracción interpersonal que ligan a los miembros. La cohesión para la tarea se relaciona con el modo en que las aptitudes y habilidades de la agrupación se conjugan para permitir un desempeño óptimo. 
Para promover la cohesión social, existen actividades para la formación de grupos con un componente de diversión o juego que son de gran utilidad; algunos ejemplos son: diseñar un logotipo u otra clase de identificación del equipo, compartir información sobre sus primeros trabajos y promover actividades que revelen las características en común de los integrantes. Por otra parte, para desarrollar la cohesión de las tareas es útil realizar actividades que les permitan a los miembros del grupo evaluar sus respectivas habilidades, fortalezas y debilidades.

La asignación de roles y normas. Con el transcurso del tiempo, todos los grupos asignan roles a sus integrantes y establecen normas, aunque esto no se discuta explícitamente. Las normas son reglas que gobiernan el comportamiento de los miembros del grupo. Atenerse a roles explícitamente definidos le permite al grupo realizar las tareas de modo eficiente. Cuando se trabaja con grupos en el aula, muchas veces los roles y las normas que rigen el desarrollo de la lección son impuestas por el docente; sin embargo, puede resultar positivo realizar actividades en las cuales se discutan y acuerden los roles y normas del grupo para garantizar su apropiación por parte de los integrantes. En este sentido, muchos docentes les proponen a los grupos que elaboren sus propias reglas o establezcan un "código de cooperación". Lo que se hace es que se les sugiere a los alumnos que identifiquen cuáles son los roles necesarios para llevar adelante un tarea y se encarguen de distribuirlos entre los miembros del equipo.

La comunicación. Una efectiva comunicación interpersonal es vital para el desarrollo de cualquier tipo de tarea. Los grupos pueden tener modos de funcionamiento que faciliten o que obstaculicen la comunicación. Se pueden realizar actividades que analicen estos modos. Algunos especialistas sugieren efectuar ejercicios en los cuales los integrantes deban escuchar a los demás y dar y recibir información.
La definición de objetivos. Es muy importante que los integrantes del equipo tengan objetivos en común relacionados con el trabajo del equipo y que cada uno pueda explicitar cuáles son sus objetivos individuales. Para ello, se sugiere asignar a los grupos recién formados la tarea de definir su misión y sus objetivos teniendo en cuenta que los objetivos compartidos son una de las propiedades definitorias del concepto equipo.

La interdependencia positiva. El aprendizaje colaborativo se caracteriza por la interdependencia positiva entre las personas participantes en un equipo, quienes son responsables de su propio aprendizaje y del aprendizaje del equipo en general. Sus miembros se necesitan unos a otros y cada estudiante aprende de los demás compañeros con quienes interactúa día a día.

Para que los integrantes tomen conciencia y experimenten lo que significa la interdependencia, ciertos docentes sugieren poner en práctica un ejercicio denominado "Supervivencia en una isla", en el que los compañeros de equipo deben imaginar cuáles son los elementos que necesitarían para sobrevivir, luego de un naufragio, en una isla desierta. En seguida, deben realizar el mismo análisis de modo grupal. En general, los rankings grupales suelen ser más precisos que la mayoría de los individuales.

La propuesta considera que estos elementos pueden ser de gran utilidad para pensar actividades tendientes a promover un verdadero trabajo en equipo en el cual el todo sea mayor que la suma de las partes. Aún así, para que esto se dé es necesario que haya un líder que sepa reflejar lo que representa el grupo y la forma en que este va a trabajar, con el fin de que sea el vocero ante las instancias superiores de la organización.

El líder es quien moldea o da forma a la estructura de cada grupo. Con su conducción se pueden formar (o no) grupos de personas que funcionen como equipos de trabajos. Este gobierna con éxito el grupo de trabajo pero no produce equipos, sino que únicamente agrupa personas para realizar una tarea. 
Huertas y Rodríguez (2006) indica que, con su accionar, el buen líder desarrolla modelos de trabajo colaborativo utilizando la mezcla adecuada de lealtad, motivación y confianza que todo ser humano necesita para creer en los objetivos grupales y ser emprendedor en beneficio de ellos. La diferencia entre un grupo de personas y un equipo de trabajo es la que determina la eficiencia de la organización. El sentimiento de pertenecer a un equipo de trabajo perdura a lo largo del tiempo $\mathrm{y}$ aunque se haya dejado de pertenecer al equipo, el sentimiento de lealtad se mantiene. Si las reglas claras conservan la amistad, entonces estas fomentan y mantienen la lealtad mutua entre el líder y los seguidores.

Hay diferentes requisitos para el establecimiento de equipos de trabajo efectivo en busca del trabajo colaborativo según el Departamento de Tecnología Educativa de la Universidad de Sevilla (2006), pues si se logra cumplir el desafío de motivar y comprometer a los participantes en la organización, surge un nuevo reto: hacer que el ingreso a equipos de trabajo sea acogedor y estimulante. Por lo tanto, es de suma importancia tomar en cuenta los siguientes elementos:

El papel de todo dirigente y de todo encargado de un equipo es generar un clima en el cual la comunicación sea fluida, se escuche a los otros y se manifiesten los desacuerdos, exista respeto entre las personas, se facilite un nivel mínimo de real comprensión por el otro y haya algún grado de afecto entre los integrantes.

Se debe propiciar las condiciones para que el equipo se concentre en la tarea y aparezca la creatividad individual y de todo el grupo, en función de lo programado.

Hay que delimitar las funciones que cumplirá cada persona, ya que es necesario dar a conocer las normas de funcionamiento, determinar cómo va a ser la dirección, establecer quién la ejercerá y, además, crear un calendario de reuniones. Asimismo, se deben respetar las funciones específicas de cada uno de los miembros.

Se tiene que establecer claramente la situación, tema o problema que se trabajará, así como preparar un programa objetivo, con una clara y precisa definición de los objetivos y las metas alcanzables.

Debe haber interés por alcanzar un objetivo común y estar de acuerdo con este tomando en consideración las motivaciones de cada miembro del grupo.

- $\quad$ Es primordial lograr un clima democrático propicio, en el cual cada persona pueda expresarse libremente sin ser juzgado por sus compañeros y en el que cada idea es aceptada por el grupo para que, por lo tanto, rechazar una idea no signifique rechazar a la persona.

En la medida que se escuchan las opiniones de todos, se obtiene la máxima información antes de decidir, pues los integrantes se convencen con argumentos.

Finalmente, para lograr para un buen trabajo en equipo es fundamental el desarrollo de la disposición a colaborar y a intercambiar conocimientos y destrezas. Esto implica contar con tiempo necesario para que cada integrante le muestre a los otros lo que sabe y esté dispuesto a facilitar los conocimientos que posee para que los demás lo aprendan.

Dentro de la propuesta, de acuerdo con el Departamento de Tecnología Educativa de la Universidad de Sevilla (2006), es tan importante conocer los aspectos que propician la formación de equipos, como saber las razones por las cuales el trabajo en equipo fracasa en muchas oportunidades, entre ellas: 


\section{A. Las metas no están claras}

Toda meta tiene que definir un objetivo. Cuando se conoce y se sabe con claridad cuál es el objetivo, es más fácil llegar a él. En los equipos de trabajo, las metas cumplen una función adicional: concentran y canalizan los aportes y la energía de cada miembro de un equipo en una sola dirección. Si no hay una meta clara, los miembros no se cohesionan y no se incrementa el desempeño del equipo. Los equipos se establecen cuando existe una meta completa y compartida.

\section{B. Hay una falta de soporte de las direcciones}

Se acostumbra tener estructuras verticales en las organizaciones; sin embargo, los equipos de trabajo se mueven horizontalmente y se usan para resolver problemas que abarcan muchas áreas y departamentos.

El trabajo en equipo es un cambio de paradigma en la organización. Esta nueva forma de trabajar puede tener éxito únicamente si las cabezas de la organización están convencidas y capacitadas para dar el ejemplo. Trabajar en equipo implica estar dispuesto a eliminar fronteras y pensar la organización como un solo bloque de trabajo.

\section{Liderazgo no efectivo de equipos}

Los miembros de equipos recién formados son como imanes del mismo polo, por consiguiente, se repelen. Se sienten incómodos porque están acostumbrados a trabajar solos y a que el éxito únicamente dependa de ellos. En esta etapa del equipo, que suele durar entre seis meses y un año, el líder debe ser directivo y demostrar fuerza para resolver conflictos, trazar metas iniciales y brindar capacitación. A medida que los miembros del equipo van adquiriendo confianza entre sí, exhiben un mayor nivel de cooperación y mejoran sus relaciones. Por ello, el líder debe dejar su estilo directivo y cambiarlo por uno participativo. Por lo tanto, el líder debe variar su estilo de liderazgo de acuerdo con el grado de madurez del equipo.

\section{Individualidad}

Un problema es limitarse a ver únicamente las diferencias e individualidades, puesto que cuando se trabaja en equipo, estas se manifiestan. Los miembros del grupo se concentran en ser únicos, en hacer las cosas "a su manera", en destacarse y en competir, lo que obstaculiza el trabajo en equipo. El individualismo ha sido fomentado por la sociedad y la empresa y, por eso, es muy difícil dejarlo de lado. Trabajar en equipo implica servir, es decir, dejar de pensar únicamente en el beneficio propio. Solo de esta forma se contribuirá a formar un verdadero equipo.

Se debe trabajar desde la administración de los centros educativos para propiciar una apertura al cambio dentro del clima escolar, la cual reconozca que es necesario modular las acciones según las necesidades espacio-temporales del entorno escolar, como lo indican Arón y Milicic (1999).

Con la implementación del trabajo colaborativo en las organizaciones, se tendrá espacio para la búsqueda de soluciones creativas y viables para las diferentes problemáticas en los centros educativos (así como para las nacionales) que rompan con ese sentido estructurado de la educación y permitan el establecimiento de un sistema horizontal.

Se replantearán proyectos a nivel de aula, como de institución en razón de lo que se vive en el contexto, con lo cual se involucrará a todas las partes en el cambio institucional.

La apertura al intercambio crítico de opiniones en busca de un cambio según el contexto permitirá el mejoramiento del 
proceso de enseñanza-aprendizaje, con lo que se cumple con la finalidad última de formación.

Se puede concluir que la propuesta se centra en un trabajo colaborativo en el cual la administración del centro escolar debe buscar la consolidación de una agrupación de personas que, trabajando juntas, compartan percepciones, tengan una propuesta en común, estén de acuerdo con los procedimientos de trabajo, cooperen entre sí, acepten un compromiso y resuelven sus desacuerdos en discusiones abiertas; esto reconociendo que lo anterior no aparece automáticamente, sino que debe irse construyendo poco a poco. La propuesta se refiere a una acción colaborativa en que la discusión no es el objetivo, sino el medio.

\section{Propuesta para el trabajo colaborativo}

\section{Objetivo general}

1. Desarrollar un sistema de aprovechamiento para el empleo correcto de los equipos de trabajo en el logro de los objetivos, dentro de los lineamientos de una adecuada gestión directiva.

\section{Objetivos específicos}

1.1 Identificar las utilidades en la organización de los equipos de trabajo.

1.2 Determinar los lineamientos oportunos para la formación de equipos de trabajo.

1.3 Formular propuestas de técnicas de trabajo en los equipos según las necesidades de la organización.

1.4 Brindarle a la organización una estructura de trabajo oportuna para el establecimiento de equipos dentro de las técnicas propuestas.

\section{Estructura y descripción de la propuesta}

Con la intención de poder lograr la implementación de una propuesta adecuada y partiendo de las lecturas de Glinz (2006), Caldeiro y Vizcarra (1999) y Robbins (1999), se requiere aclarar lo que se entiende como técnica para, de esta forma, dar un mayor sentido de claridad al trabajo.

En este caso, las técnicas son:

- Los procedimientos necesarios para buscar las soluciones, optimizar la mejor de ellas y decidir cuál es la más apropiada, luego de haber identificado el problema.

- Los caminos que orientan al equipo sobre cómo trabajar e indican la ruta a seguir, pues facilitan la consecución de los objetivos.

- Las maneras, procedimientos o medios sistematizados de organizar y desarrollar las actividades del equipo.

- Los medios o los métodos empleados en situaciones grupales que buscan la interacción de todos los miembros de un equipo a fin de lograr los objetivos planteados.

Dentro de la propuesta que se desea implementar, hay diversas técnicas que sirven como apoyo para ella y que, de acuerdo con lo analizado dentro del proceso de investigación, se adaptan a las necesidades presentadas por las organizaciones, según lo demostrado por la investigación y los requerimientos de los centros escolares.

En esta propuesta para implementar el trabajo colaborativo, se hace necesario que la administración del centro escolar tome las medidas anteriormente mencionadas y las introduzca gradualmente según diferentes técnicas precisas para ella, como ha sido detallado en el estudio realizado.

Algunas de estas técnicas son: 
1) Técnica interrogativa o de preguntas

2) Mesa redonda

3) Seminario

4) Estudio de casos

5) Foro.

Dichas técnicas deben ser evaluadas por cada uno de los administradores de la educación y ser llevadas a la contextualización del caso para procurar adaptarlas tomando en consideración las necesidades y requerimientos de la organización. Es oportuno promover la creatividad en dichas técnicas partiendo de los intereses de los miembros y los objetivos por alcanzar.

Para poder hacer una ruptura de paradigmas en las organizaciones educativas con la implementación del trabajo colaborativo, se debe hacer una diferenciación entre la tecnología y la técnica, pues la tecnología está ligada al uso y a la producción, mientras que la técnica, por su parte, está mucho más cerca del ser humano y por consiguiente, del trabajo colaborativo. Este es una muestra del conjunto de esfuerzos en función del cumplimiento de los objetivos y una acción que potencia las capacidades humanas.

Las técnicas mencionadas, que demuestran las formas de inclusión del trabajo colaborativo, son importantes en tanto se responda a las concepciones sociológicas, en cuyo planteamiento el conocimiento es el producto de la acción grupal y no de la persona concreta, motivo por el cual se afirma la supremacía de la opinión grupal.

En el trabajo colaborativo, las técnicas se resuelven con una base democrática, es decir, con el voto de la mayoría. A partir de ello se puede decir que todo conocimiento surge o se construye a partir de interacciones entre los miembros de la organización y su entorno.

A medida que las técnicas propicien la consecución de objetivos de alto nivel de importancia para la organización, como la creatividad y la solución de problemas, los miembros del centro educativo favorecerán un clima organizacional donde son ellos quienes participan en la toma de decisiones, eligen las estrategias a seguir para la solución de problemas, determinan qué tan correcta o incorrecta es la respuesta fundamentada en datos producidos y validados por ellos y, además, están involucrados en determinar sus propias metas y los medios para evaluar el logro de éstas.

Con las presentes técnicas se promueve romper con el sistema estructuralista que manejan las organizaciones educativas, producto de las políticas educacionales trazadas para definir las metas de la educación costarricense. Se busca lograr un sistema de métodos colaborativos, en el cual se propicien facilitadores que permitan que todos estén al mismo nivel, sin las barreras y distancias que produce la falta de valores inherentes al equipo de trabajo.

\section{Recursos ineludibles para las técnicas de la propuesta}

\section{A. Recursos humanos}

- Director o directora de la organización

- Docentes del centro educativo

- Personal administrativo de la organización

Invitados especiales a la técnica

\section{B. Recursos financieros}

Aportes de la Junta de Educación y el Patronato Escolar Talleres creados por autogestión Aportes de entidades externas

\section{Recursos tecnológicos}

Equipo de audio

Equipo de video

Retroproyector 


\section{Evaluación de las diferentes técnicas}

Según como se desarrolle el cronograma escolar, de igual forma se trabajarán las diferentes técnicas, de acuerdo con las disposiciones de la dirección del centro educativo y las facilidades temporales. Es propicio considerar que se requiere al menos una tarde por técnica, para desarrollarla adecuadamente y que ésta no se vea interrumpida por factores de tiempo.

Toda actividad por desarrollar debe ir acompañada de un adecuado proceso de planificación, además del compromiso de participación del personal, para asegurar su éxito.

Las técnicas deberán ser evaluadas con un instrumento previamente elaborado, el cual será llenado por los sujetos cuando finalice el taller. Se contará con espacios para conversar $\mathrm{y}$, en un intercambio dinámico, se reconocerá cuáles factores deben o puede mejorarse según lo vivido y expresado por los partícipes. El objetivo es que se dé una adecuada evaluación de las técnicas aplicadas, en las que se determinará la interacción entre las personas. Además, se irán evaluando y ponderando los resultados positivos y negativos.

\section{Establecimiento de las condiciones básicas de las técnicas para la propuesta}

Dentro de los requerimientos necesarios para el adecuado desarrollo de las técnicas, se pueden enumerar algunos elementos generales para el éxito de los diferentes talleres, al reconocer que, si en algún momento surge la necesidad de algún elemento específico, esto es propio de la dinámica de las actividades, de acuerdo con las variables que se presenten durante su ejecución.

Se pueden establecer los siguientes elementos como necesarios:

- Un espacio amplio, que reúna las condiciones adecuadas para el trabajo prolongado en forma grupal.
- Sistemas electrónicos de sonido que permitan establecer adecuados procesos de comunicación en espacios abiertos y con amplia participación.

- Hojas y papeles oportunamente seleccionados según las dinámicas de trabajo.

- $\quad$ Equipo de cómputo para la preparación de los diferentes materiales.

- Disponibilidad de tiempo por parte de los participantes.

- Acceso oportuno a la bibliografía pertinente para cada taller.

\section{Referencias bibliográficas}

Alfaro, B. (1999). Funciones interpersonales en la gestión administrativa. Tesis de maestría sin publicar, Universidad de Costa Rica, San Ramón, Alajuela, Costa Rica.

Arón, A., y Milicic, N. (1999). Clima social escolar y desarrollo personal. Un programa de mejoramiento. Santiago, Chile: Editorial Andrés Bello.

Caldeiro, G., y Vizcarra, M. (1999). El aprendizaje por trabajo cooperativo. Consultado el 16 de enero del 2007 de: http://educacion.idoneos. com/index.php/El_aprendizaje_ por trabajo $_{-}$cooperativo

Chiavenato, I. (2002). Gestión del talento humano. Colombia: McGraw-Hill Interamericana, S.A.

De Benito, B. (1999). Taller: Redes y trabajo colaborativo entre profesores. Consultado el 3 de marzo del 2007, de: http://gte.uib.es/articulo/ EDUTEC99.pdf

España, Universidad de Sevilla, Departamento de Tecnología Educativa. (2006). Trabajo colaborativo. Conceptualización. Consultado el 3 de marzo del 2007, de: 
http://tecnologiaedu.us.es/cursobscw/ apartados/apartado11.htm\#visto

Glinz, P. (2006). Un acercamiento al trabajo colaborativo. Consultado el 3 de marzo del 2007, de: http://www.rieoei. org/deloslectores/820Glinz.PDF
Huertas, J., y Rodríguez, G. (2006). Desarrollo de habilidades directivas. México: Pearson Education.

Robbins, S. (1999). Comportamiento organizacional. México: Prentice Hall. 
